\title{
Uveal Melanoma cM1 TNM Finding v8
}

National Cancer Institute

\section{Source}

National Cancer Institute. Uveal Melanoma CM1 TNM Finding v8. NCI Thesaurus. Code C140607.

Uveal melanoma with distant metastasis. (from AJCC 8th Ed.) 